\title{
Inhibition of invasiveness and expression of epidermal growth factor receptor in human colorectal carcinoma cells induced by retinoic acid ${ }^{1,2}$
}

\author{
SUN-BAODONG, JINDAN SONG ${ }^{3}$. \\ Key Laboratory of Cell Biology, Ministry of Public Health \\ of China, China Medical University, Shenyang 110001, \\ China.
}

\section{ABSTRACT}

Human amniotic basement membrane (HABM) model and agarose drop explant method were used to investigate the effects of retinoic acid ( RA ) on the invasiveness and adhesiveness to the basement membrane,and the migration of a highly invasive human colorectal cancer cell line CCL229. Results showed that 5 x $10^{6}$ MRA markedly reduced the in vitro invasiveness and adhesiveness to the HABM, and the migration of the CCL229 cells. In addition, to elucidate the relation between expression of epidermal growth factor receptor (EGFR) and the invasiveness of the colorectal carcinoma cells, two well-differentiated, but with different invasiveness colorectal cancer cell lines were compared at mRNA level for expression of EGFR by using EGFR cDNA probe labeled with digoxigenin (DIG). Expression of EGFR was shown to be markedly higher in the highly invassive CCL229 cells than that in the low invasive CX-1 cells. Furthermore, expression of EGFR in RA treated CCL229 cells gradually decreased with time, the level being the lowest on day 6 of the RA treatment.

Key words: Retinoic acid, colorectal carcinoma cell, invasiveness, epidermal growth factor receptor, inhibition.

\footnotetext{
1. Dedicated to 80th anniversary of Professor Zhen YAO.

2. This work was supported by National Natural Science Foundation.

3. Corresponding author.
} 
Drug inhibition of invasiveness and expression of EGFR

\section{INTRODUCTION}

As a derivative of vitamin A, RA can inhibit the proliferation of many malignant cells and elicit differentiation in some tumor cells[1-5] . Recent studies have shown that RA modulates synthesis of over 40 proteins through its nucleic receptor[6]. For instance, RA induces the synthesis of fibronectin (FN) in certain tumor cells and activates the gene coding for $\mathrm{B} 1$ subunit of laminin (LN) causing its expression[7, 8]. FN and LN are known to be the most important components of non-collagen glycoproteins in the extracellular matrix and are closely related to the invasion and metastasis of the cancer cells. There also have been evidence that RA inhibits the expressions of collagenase and stromelysin in some cells[9-11], and both of these two proteolytic enzymes are often overexpressed in invasive cells. These results indicate that RA may inhibit invasion of cancer cells. Franker[12] and Nakajima[10] independantly reported the inhibitive effects of RA on the metastasis of human breast cancer cell and invasion of rat mammary cancer cell to basement membrane. There has been no reports so far, however, on the effects of RA on the invasiveness of colorectal cancer.

EGFR is a single-chain transmembrane glycoprotein on the cell surface which possesses tyrosine kinase activity. The sequence of its intracellular portion is $90 \%$ homologous to that of the protein coded by v-erb $\beta$ oncogene. EGFR is expressed in many cancer cells with various degrees[13-15], and is closely associated with the invasion and metastasis of some cancer cells. It is now confirmed that expression of EGFR is positively correlated to the invasiveness of human breast cancer[16], bladder cancer[17], and gastric carcinoma cells[18]. Yasui and associates[18] demonstrated higher expression of EGFR in human colorectal cancer cells than that in the normal colorectal tissue. Bradley et a1[19] further confirmed that well-differentiated colorectal carcinoma had markedly higher EGFR contents on cell surface than the poorly differentiated, but the relation between expression of EGFR and invasion of the carcinoma was inconclusive. Using two well-differentiated colorectal cell lines but with different invasiveness, CCL229 (highly invasive) and CX-1 (low invasive), we investigated the differential expressions of EGFR in the two cell lines, and comparatively studied their invasiveness in vitro, related biologic properties, and EGFR expression prior to and after treatment with RA.

\section{MATERIAL AND METHODS}

\section{Cells and cell culture}

The two human colorectal carcinoma cell lines CCL229 and CX-1 were generous gifts of DanaFarber Cancer Institute of Harvard Medical School, USA. The cell lines, maintained in Dulbecco's modified Eagle's medium (GIBCO, BRL) and supplemented with $10 \%$ calf serum, were grown in a humidified atmosphere of $95 \%$ air $/ 5 \% \mathrm{CO}_{2}$ at $37^{\circ} \mathrm{C}$.

\section{Treatment of RA on CCL229 cells}

CCL229 cells at exponential growth stage were subcultured and randomly allocated into exper- 
imental group and control group. The experimental group was added with All-trans-RA (SIGMA) dissolved in absolute ethanol to a final concentration of $5 \times 10^{-6} \mathrm{M}$; the control group was added with the same amount of absolute ethanol. Both cell groups were cultured for $72 \mathrm{~h}$ in humidified $95 \%$ air $/ 5 \% \mathrm{CO}_{2}$ at $37^{\circ} \mathrm{C}$.

\section{Inhibitive effects of $R A$ on in vitro invasiveness of CCL229 cells}

HABM model established by Liotta et a1[20] was used. Cells were resuspended in DMEM media containing RA (final concentration $5 \times 10^{-6} \quad M$ ) supplemented with $5 \%$ calf serum and were applied in the upper compartment; the lower compartment was filled with DMEM free from calf serum. The cells were cultured for 48, 72, 96 hours respectively in $95 \%$ air $/ 5 \% \mathrm{CO}_{2}$ at $37^{\circ} \mathrm{C}$. The nucleopore filter $(0.45 \mu \mathrm{m}$ pore size $)$ tightly attaching to the matrix surface of the amnion was gently removed, fixed, and stained with H.E. Cells adhering to the filter membrane were counted under a light microscope. Total number of cells were counted in randomly selected 10 low power fields and the procedure was repeated 4 times.

\section{Effects of RA on migration of CCL229 cells}

The agarose drop explant method[21] was adopted, and the procedure was repeated 4 times.

\section{Adhesiveness of RA treated CCL229 cells to the basement membrane}

Cells were seeded onto the upper compartment of HABM model $\left(1 \times 10^{5}\right.$ cells $\left./ \mathrm{ml}\right)$, and were maintained for 2,4, and $6 \mathrm{~h}$ respectively in $95 \%$ air $/ 5 \% \mathrm{CO}_{2}$ at $37^{\circ} \mathrm{C}$. Supernatant of the media was withdrawn. Adhering cells were washed 3 times with PBS and collected after digestion with $0.25 \%$ trypsin / $0.02 \%$ EDTA. Cells were counted and the adhesion rate was calculated. The process was repeated 4 times[22].

\section{Effects of RA on surface morphology of CCL229 cells}

Cells treated with RA for $72 \mathrm{~h}$ were seeded on glass coverslips, and were observed with conventional scanning electron microscopy.

\section{Extraction of RNA}

RNAs of CCL229 cells, CX-1 cells, and CCL229 cells treated with RA for 2, 3, 4, 5, and $6 \mathrm{~d}$ in experimental and control groups were extracted using the method established by Chen Yuhua and Song jindan[23]. $\mathrm{OD}_{260 / 280}$ values were obtained with ultraviolet spectrophotometry, and the quantitation of RNA was carried out at $\mathrm{OD}_{260}$.

\section{Labeling of cDNA probe of human EGFR and detection by hybridization}

DIG DNA Labeling and Detection kit was a product of Boehring Manaheim Biochemica. EGFR cDNA probe was purchased from Yuanping Biotechnology Company, Beijing. EGFR cDNA probe is labeled by random primed incorporation of digoxigenin-labeled dUTP. Measurment of mRNA activity was carried out with conventional dot hybridization[24].

\section{RESULT}

\section{Inhibition of in vitro invasiveness of CCL229 cells by RA}

The number of cells which penetrated the amnion in the group treated with RA $\left(5 \times 10^{-6} M\right)$ was $40 \%$ of that of the control group at $48,72,96$ h respectively indicating the inhibitive effects of RA on in vitro invasiveness of CCL229 cells $(\mathrm{P}<$ 0.01) (Fig 1). 
Drug inhibition of invasiveness and expression of EGFR

Effects of RA on the migration of CCL229 cells

The migration of cancer cells was assessed by the number of cells which migrated out of the agarose drops and the leading edge of the migration during a certain time interval. The number of cells that moved out of the drops in the group treated with RA were about half of those in the control group $(\mathrm{P}<0.01)$, and the leading edge in the experimental group was also significantly shorter than that in the controls $(\mathrm{P}$ $<$ '0.01) (Tab 1).

Tab 1. Effect of RA on migration of CCL229 cells.

\begin{tabular}{ccccc}
\hline & \multicolumn{2}{c}{ Cell Number $(\mathrm{x} \pm \mathrm{SD})$} & \multicolumn{2}{c}{ Leading Edge $(\mu \mathrm{m})(\mathrm{x} \pm \mathrm{SD})$} \\
\hline & Control & +RA & Control & $+\mathrm{RA}$ \\
\hline $24 \mathrm{~h}$ & $13 \pm 3$ & $5 \pm 1$ & $35.5 \pm 8.4$ & $18.7 \pm 1.5$ \\
$48 \mathrm{~h}$ & $22 \pm 4$ & $10 \pm 3$ & $64.1 \pm 10.2$ & $36.6 \pm 8.5$ \\
$72 \mathrm{~h}$ & $56 \pm 8$ & $24 \pm 5$ & $90.3 \pm 15.4$ & $58.3 \pm 11.8$ \\
\hline
\end{tabular}

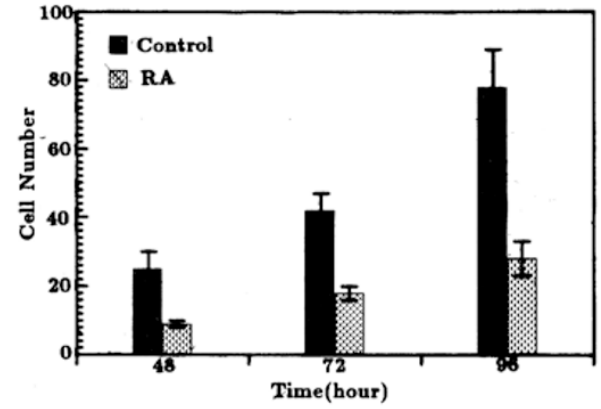

Fig 1. Effect of RA on invasion of CCL229 cells.

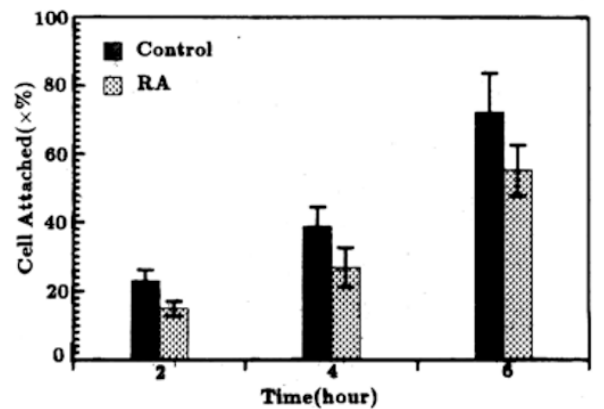

Fig 2. Effect of RA on adhesion of CCL229 cells.

Effects of RA on the adhesiveness of CCL229 cells

The adhesiveness of the cancer cells was evaluated by the adhesion rate of the cells to the HABM. Fig 2 showed that the adhesiveness of CCL229 cells treated with RA was near $30 \%$ less than that of the controls $(\mathrm{P}<0.01)$.

Effects of RA on surface morphology of CCL229 cells

Scanning electron microscopy showed that CCL229 cells not treated with RA assumed a fusiform shape with strong anchorage. Many microspikes and filopodia were revealed on the surface (Fig 3A). After treatment with RA, processes on the cell surface significantly diminished, and the microspikes and filopodia virtually disappered (Fig 3B).

Expression of EGFR in the colorectal carcinoma cells

Dot hybridization with DIG labeled EGFR cDNA probe showed that expression of EGFR in the highly invasive CCL229 cells was markedly higher than that in the 
low invasive CX-1 cells (Fig 4). The expression of EGFR in CCL229 cells treated with RA $\left(5 \times 10^{-6} \mathrm{M}\right)$ gradually decreased with time, and was hardly detectable on day 6 (Control not shown).
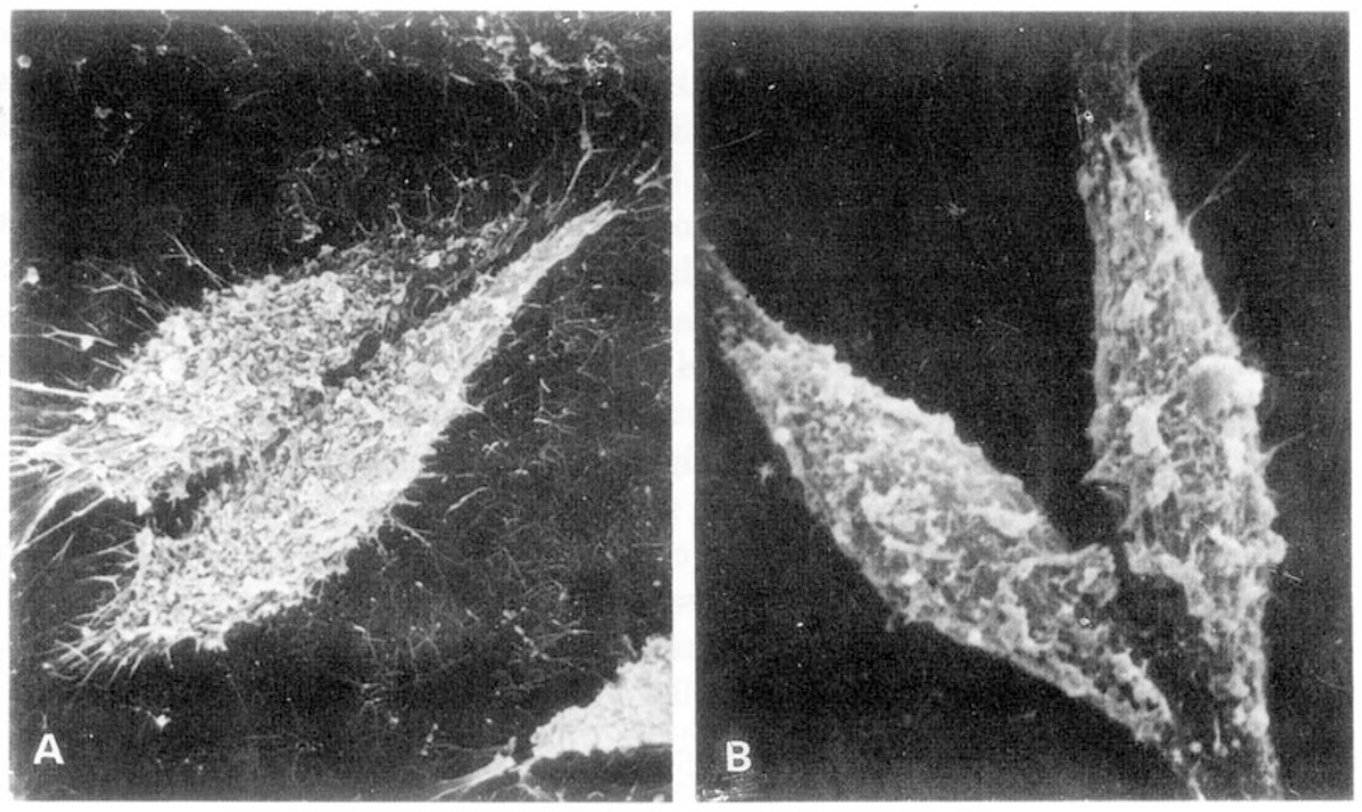

Fig 3. SEM micrograph of CCL229 cells: (A) without RA treatment, $\times 1800$; (B) with RA treatment, $\times 2000$.

1

2

$\mathbf{3}$

4

A

B

Fig 4. Expression of EGFR in human colorectal carcinoma cells. (A1, A2, A3, A4, B4 corresponding to the CCL229 cells on 2, 3, 4, 5, 6 day after RA treatment respectively, control not shown; B1, B2 indicate GLL229 and CX-1 cells without RA treatment respectively. $1.8 \mu \mathrm{g}$ RNA each sport.))

\section{DISCUSSION}

The primary factors which influence the invasiveness of cancer cells are their migration, adhesiveness to the basement membrane and the capacity to secrete pro- 


\section{Drug inhibition of invasiveness and expression of EGFR}

teolytic enzymes to degrade the extracllular matrix[25]. Our previous study on the comparison of two well differentiated cell lines: CCL229 and CX-1 cells showed that invasiveness, adhesiveness to the basement membrane, as well as the migration of CCL229 cells were significantly higher than those of CX-1 cells, respectively. The morphology of the highly invasive CCL229 cells was characterized by numerous microspikes and filopodia on the cell surface[26]. These results indicated that invasiveness of cancer cells was consistent with their adhesiveness and migration, and was correlated to the morphology of the cell surface. The present study demonstrated that $5 \times 10^{-6} \mathrm{M}$ RA had obvious inhibitive effects on the in vitro invasiveness, adhesiveness and migration of the CCL229 cells, and markedly reduced the microspikes and filopodia on the cell surface. Although RA could affect the invasivehess through reducing the migration and adhesiveness to the basement membrane, the most crucial factor causing the penetration of the amnion rested on the capacity of the cancer cells to secrete proteolytic enzymes that degraded the basement membrane and matrix. There have been reports that RA can inhibit the expression of collagenase and stromelysin in some cancer cells[9-11]. These two are the most important proteolytic enzymes synthesized and secreted by the endoplasmic reticulum, and play a crucial role in the invasion by cancer cells. Further investigations have shown that the promoters of the collagenase and stromelysin genes contain the binding sites for transcription factor AP1, and RA acts through its nuclear receptor to reduce the activity of transcription factor AP1 and thus inhibit the expression of the two enzymes[27]. It was also reported that RA can inhibit the metastasis of human breast carcinoma and invasion of rat mammary carcinoma to the basement membrane[10,12]. There has been no published study, however, on the effects of RA on invasiveness of colorectal carcinoma.

The over-expression of EGFR in some cancers has received much attention. It appears that expression of EGFR might be used as a marker for prognosis. Salnsburg[16] first noted that expression of EGFR in highly invasive human breast carcinoma cells was higher than that in the benign tumors. Neal et al[17] confirmed that EGFR was overexpressed in human invasive bladder cancer cells. Yasui et al[18] observed that EGFR in metastatic carcinomas in perigastric lymph nodes was markedly higher than that of primary carcinomas, and the expression of EGFR was correlated to invasion, metastasis, and prognosis of gastric carcinoma. However, the relation between expression of EGFR and invasiveness of colorectal carcinoma remains obscure. The observation that EGFR are differentially expressed in colorectal carcinoma cells with different degree of differentiation[19] has prompted us to compare the EGFR expression of two cell lines which are both well differentiated but have very different invasiveness. Our results showed that expression of EGFR in the very invasive CCL229 cells is significantly higher than that in the low invasive CX-1 cells. In addition, expression of EGFR in CCL229 cells was down regulated as the invasiveness of CCL229 cells became decreased during the induction by $\times 10^{-6} \mathrm{M}$ RA. These results all together indicate that invasiveness of colorectal carcinoma 
cells may be consistent with the degree of EGFR expression. There are similar reports demonstrating that RA may inhibit expression of EGFR in some other cancer cells. Zheng et al[28] found that RA inhibited EGFR expression in human epidermoid cancer cell line ME180 and that the inhibitive effect was most evident when the concentration of RA was $10^{-5} M$. They also showed that RA suppressed EGFR gene transcription and mRNA levels by its nuclear receptor. Another study reported that RA decreased mRNA levels of EGFR by 2- to 4 -folds in cultured human cerebrocervical squamous carcinoma cells [29]. To our knowledge, our study is the first report on the inhibition by RA of expression of EGFR in colorectal carcinoma cells.

In conclusion, our study demonstrated that the expression of EGFR is higher in highly invasive colorectal carcinoma cells than that in low invasive colorectal carcinoma cells; and the decrease of the expression of EGFR in a time- and dosedependent manner after the cells are exposed to RA is accompanied by a synchronous reduction of invasiveness of the caner cells.

\section{REFERENCES}

[1] Moon RC, McCormick DL, Mehta RG. Inhibition of carcinogenesis by retinoids. Cancer Res 1983; 43:2469-75.

[2] Liu Youhua,Wang Yunqing. Studies on microscopic and submicroscopic structure of human promyelocytic leukemia cells (HL- 60) during differentiation induced by retinoids and dimethyl sulfoxide. Acta Biologiae Experimentalis Sinica 1985; 18(4):389-404.

[3] Lotan R. Effects of vitamin A and its anologs (retinoids) on normal and neoplastic cells. Biochim Biophys Acta 1980; 605:33-91.

[4] Brown R. Retinoids alter the direction of differentiation in primary cultures of cutaneous keratinocytes. Differentiation 1985; 28:268.

[5] Sherman ME. Retinoids and Cell Differentiation. Boca Raton FL: CRC Press Inc 1986.

[6] Boyd AS. An overview of the retinoids. Am J Med 1989; 86:568-574.

[7] Yao Zhaowei, Zha Xiliang, Ye Jiannan, Chen Huili. Some reverse effects of retinoic acid on the membranous phenotype of human hepatocarcinom a cell line. Chinese Journal of Biochemistry and Biophysics 1990; 22:147-51.

[8] Vasios GW, Gold JD, Patkovich M, Chambon P, Gudas LJ. A retinoic acid- responsive element is present in the 5'flanking region of the laminin B1 gene. Proc Natl Acad Sci USA 1989; 86:9099-103.

[9] Nakajima M, Lotan D, Baig M, Lotan R. Inhibition by retinoic acid of type IV collagenolytic metalloproteinase production in metastatic rat mammary adenocarcinoma cells. J Cell Biol 1987; 105(4, part. 2):217a.

[10] Nikajima M, Lotan D, Baig MM, et al. Inhibition by retinoic acid of type IV collagenolysis and invasion through reconstituted basement membrane by metastatic rat mammary cancer. Cancer Res 1989;49:1698-706.

[11] Nicholson RC,Mader S, Nagpal S, Leid M, Rochette-Egly C, Chambon P. Negative regulation of the rat stromelysin gene promoter by retinoic acid is mediated by an AP1 binding site. EMBO J 1990; 9:4443-54.

[12] Fraker LD, Halter SA, Forbes JT. Growth inhibition by retinol of a human breast carcinoma cell line in vitro and in athymic mice. Cancer Res 1984; 44:5757-63.

[13] Hunts J, Ueda M, Ozawa S, Abe o,Pastan L, Shimizu N. Hyperproduction and gene amplification of the epidermoid growth factor in squamous cell carcinomas. Jpn J Cancer Res 1985; 76:663-6. 


\section{Drug inhibition of invasiveness and expression of EGFR}

[14] Veale D, Marsh C, Ashcroft T, Harris AL. Epidermal growth factor receptors in non-small cell lung cancer. Br J Cancer 1985; 52:441.

[15] Korc M, Meltzer P, Trent J. Enhanced expression of epidermal growth factor receptor correlates with alternations of chromosome 7 in human pancreatic cancer. Proc Natl Acad Sci USA 1986; 83:5141-4.

[16] Salnsbury JRC, Farndon JR, Harris AL, Sherbet GV, Sherbet GV. Epidermal growth factor receptors on human breast cancers. Br J Surg 1985; 72:186-8.

[17] Neal DE, Marsh C. Epidermal growth factor receptors in human bladder cancer: comparison of invasive and superficial tumours. Lancet $1985 ; \mathbf{1}: 366-8$.

[18] Yasui W, Sumiyoshi H, Hata J, et al. Expression of epidermal growth factor receptor in human gastric and colonic carcinomas. Cancer Res 1988; 48:137-41.

[19] Bradley S J, Garfinkle G, Walker E, Salem R, Chen LB, Steel G. Increased expression of the epidermal growth factor on human colon carcinoma cells. Arch Surg 1986; 121:1242-7.

[20] Liotta LA, Lee CW, Morakis DJ. New method for preparing large surfaces of intact human basement membrane for tumor invasion studies. Cancer Lett 1980; 11:141-52.

[21] Varanl J, Orr W, Ward PA. A comparison of the migration patterns of normal and malignant cells in two assay systems. Am J Pathol 1978; 90:159-71.

[22] Murray JC, Liotta LA, Rennard SI, Martin GR. Adhesion characteristics of murine metastatic and nonmetastatic tumor cells in vitro. Cancer Res 1980; 40:347-51.

[23] Chen Yuhua, Song Jindan. A simple, easy and practical method for the preparation of RNA. Chinese Medical Biology Research. Cheng Du: Si Chuan Science and Technology Publishing House. 1995:235.

[24] Sambrook J, Fritsch T, Maniatis T. Molecular cloning. A Laboratory manual. 2nd ed. Cold Spring Harbor Laboratory Press 1989; 372-3.

[25] Liotta LA. Cancer cell invasion and metastasis. Scientific American 1992; February:34-41.

[26] Sun Baodong, Song Jindan. In vitro comparative study on the invasive abiligy and its related biological properties of different colorectal caroinmoma cell lines. (to be published).

[27] Schule R, Rangarajan P, Yang N, et al. Retinoic acid is a negative regulator of AP- l-responsive genes. Proc Natl Acad Sci USA 1991; 88:6092-6.

[28] Zheng ZS, Goldsmith LA. Modulation of epidermal growth factor receptors by retinoic acid in ME180 cells. Cancer Res 1990; 50:1201-5.

[29] Lei Wei, Wu Min. Molecular mechanism of the regulation of retinoic acid in proliferation and differentiation. Foreign Medical Molecular Biology: subunit. 1994; 16(2):83-6.

Received 6-7-1995. Revised 12-7-1995. Accepted 14-7-1995. 